\title{
Inverse and normal coronal mass ejections: evolution up to 1 AU
}

\author{
E. Chané, B. Van der Holst, C. Jacobs, S. Poedts, and D. Kimpe
}

Centrum voor Plasma-Astrofysica, KU Leuven, Celestijnenlaan 200B, 3001 Leuven, Belgium

e-mail: Emmanuel. Chane@wis . kuleuven. be

Received 8 July 2005 / Accepted 20 September 2005

\section{ABSTRACT}

\begin{abstract}
Simulations of Coronal Mass Ejections (CMEs) evolving in the interplanetary (IP) space from the Sun up to 1 AU are performed in the framework of ideal magnetohydrodynamics (MHD) by the means of a finite volume, explicit solver. The aim is to quantify the effect of the initiation parameters, such as the initial magnetic polarity, on the evolution and on the geo-effectiveness of CMEs. The CMEs are simulated by means of a very simple model: a high density and high pressure magnetized plasma blob is superposed on a background steady state solar wind model with an initial velocity and launch direction. The simulations show that the initial magnetic polarity substantially affects the IP evolution of the CMEs influencing the propagation velocity, the shape, the trajectory and even the geo-effectiveness.

We also tried to reproduce the physical values (density, velocity, and magnetic field) observed by the ACE spacecraft after the halo CME event that occurred on April 4, 2000.
\end{abstract}

Key words. solar wind - Sun: coronal mass ejections (CMEs) - magnetohydrodynamics (MHD)

\section{Introduction}

During October and November 2003, a series of solar eruptions occurred and gave rise to some of the largest geomagnetic storms of the solar cycle. During this period, $59 \%$ of the reporting spacecrafts experienced some effects from the solar activity (Gopalswamy et al. 2005). Geomagnetic storms can perturb and interrupt electric power equipment, cause radio blackouts and navigation system failures, and damage satellite systems. Surprisingly, it seems that sperm whale strandings are also related to solar activity (Vanselow \& Ricklefs 2005). In order to improve geomagnetic storm predictions, studies on CME evolution are important.

Gombosi et al. (2000) numerically simulated a CME propagating from the Sun to the Earth interacting with the magnetosphere-ionosphere system. Manchester et al. (2004a) then performed the same kind of simulation but with a Gibson and Low CME model in a first step up to $32 R_{\odot}$, and later up to 1 AU (Manchester et al. 2004b). More recently Lugaz et al. (in press) used the data from a numerical simulation to reproduce line of sight images or even to give a stereographic view of CMEs as it would be observed by the Solar Terrestrial Relations Observatory (STEREO) project.

Numerical simulations of CMEs are widely using different background wind and CME models so that it becomes difficult, to compare the results of different authors. Jacobs et al. (2005) made a study on the effect of the applied background wind model on the IP CME evolution. Chané et al. (2005) extended this study and showed the importance of the magnetic polarity of the initial CME flux rope on the evolution of the CME. Unfortunately, in these simulations the computational domain stopped at $30 R_{\odot}$ due to lack of CPU power. In the present paper, however, the computational domain is extended up to $1 \mathrm{AU}$. This enables us to produce in situ data close to the Earth in order to fully investigate the influence of the initial magnetic polarity on the geo-effectiveness of the CMEs.

The applied wind model is presented in Sect. 2. In Sect. 3, the CME initiation is explained (Sect. 3.1) and the ICME simulations are presented. The important characteristics are explained and discussed for the inverse and for the normal CMEs. In Sect. 4 we model the halo CME event that occurred on April 4, 2000.

\section{The solar wind model}

As background solar wind model we use a reconstruction of the MHD wind model used and described by Manchester et al. (2004a), where the full set of ideal MHD equations is solved. The correct velocity ratio (polar/equator) is obtained by means of an extra heating source term which, in normalized form, is given by:

$Q=\rho q_{0} \exp ^{-\frac{(r-1)^{2}}{\sigma^{2}}}\left(T_{0}-\gamma \frac{p}{\rho}\right)$,

where the profiles of both $T_{0}$ and $\sigma$ have a $\theta$ - and an $r$-dependence. The resulting $\theta$-profiles of the solar wind density and radial velocity at $1 \mathrm{AU}$ are shown in Fig. 1 . The main characteristics of this solar wind model at $1 \mathrm{AU}$ are summarized 

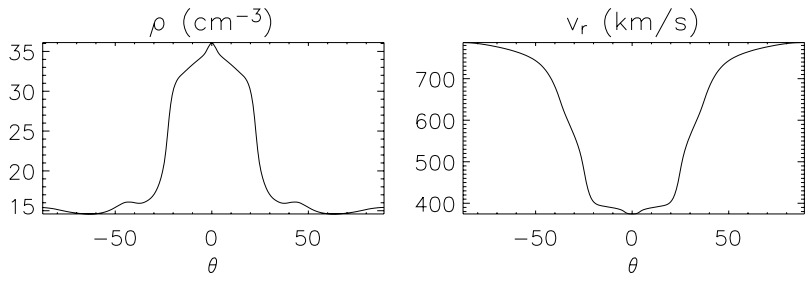

Fig. 1. The $\theta$-profiles of the solar wind density and radial velocity at $1 \mathrm{AU}$.

Table 1. Wind model characteristics ar 1 AU.

\begin{tabular}{ll}
\hline \hline \multicolumn{1}{c}{ Wind model } \\
\hline Density $\left[\mathrm{m}^{-3}\right]$ & $1.55 \times 10^{7}$ \\
$\quad$ Pole & $3.62 \times 10^{7}$ \\
Equator & 0.43 \\
$\quad$ Ratio & \\
Velocity $\left[\mathrm{km} \mathrm{s}^{-1}\right]$ & 787 \\
$\quad$ Pole & 374 \\
Equator & 2.1 \\
$\quad$ Ratio & \\
Temperature $[\mathrm{K}]$ & $0.068 \times 10^{6}$ \\
$\quad$ Pole & $0.022 \times 10^{6}$ \\
$\quad$ Equator & 3.14 \\
Ratio & \\
Magnetic field $[\mathrm{G}]$ & $5.47 \times 10^{-6}$ \\
Pole & $1.05 \times 10^{-8}$ \\
Equator & 519 \\
Ratio & $0.86 \times 10^{-13}$ \\
Total mass flux $\left[M_{\odot} / \mathrm{yr}\right]$ & \\
\hline
\end{tabular}

in Table 1. Our model is an axisymmetric 2.5D wind model (i.e. we consider three vector components that depend on only two spatial coordinates) that includes differential rotation. The solar rotation is incorporated at the inner boundary and leads to the formation of the Parker spiral in the IP space. Because we use a $2.5 \mathrm{D}$ model, we have to assume that the magnetic and the rotational axis of the sun are not tilted. Nevertheless, this assumption has a minor influence on the solar wind model. In comparison, the choice of the source term is more crucial in order to produce a good wind, i.e. a wind with the correct observational parameters.

\section{ICME simulations}

\subsection{CME initiation}

Klimchuk (2000) reviewed the many different theoretical models for CME initiation and distinguished two types of models based on basic physical properties, such as energetics, structure and dynamics, viz. directly driven models and storage and release models. However, all these models have difficulty explaining one or more aspects of observations and, hence, the present models need to be improved. Nevertheless, CME shock evolution studies very often apply simplified generation models for the CMEs, e.g. "density-driven" models consisting of a high-density (and high-pressure) plasma blob superposed on a solar wind (Groth et al. 2000) or CMEs generated by imposing an extra mass flow (Keppens \& Goedbloed 1999).

In order to study the propagation of fast CMEs and CME shocks in the interplanetary space from the solar corona up to $1 \mathrm{AU}$, a very simple model is used to generate the CMEs in the present paper. This simple model gives us an easy way to study the importance of the initial magnetic polarity of the CME. A high density and high pressure magnetized plasma blob is superposed on the background steady state solar wind model. The CMEs are launched at a certain velocity, in a given direction and are further characterized by a given density, magnetic field strength and magnetic polarity. The initial CME magnetic field and the background wind magnetic field can have the same or the opposite polarity. We call it respectively an inverse and a normal CME, according to the nomenclature of Low \& Zhang (2002) (see Chané et al. 2005, for more details about the CME initiation).

The radius of the initial plasma blob is $0.29 R_{\odot}$ and its center is located at $1.5 R_{\odot}$. In the center of the initial perturbation, the velocity is about $1000 \mathrm{~km} \mathrm{~s}^{-1}$ and the density is 5 times higher than the density on the surface of the Sun. In the initial plasma blob, the maximal magnetic field strength is $\sim 3.44 \mathrm{G}(\sim 0.344 \mathrm{mT})$. In all the numerical simulations discussed in this section, the CMEs have the same initial density, pressure, velocity and magnetic strength. The only difference will be the initial magnetic polarity: inverse or normal. In the following subsections, the influence of the initial magnetic polarity on the time of arrival, on the evolution path and on the geo-effectiveness of the ICMEs will be investigated.

\subsection{Characteristics of the CMEs}

In this subsection, we discuss the characteristic shape (density redistribution and magnetic field configuration) of the normal and the inverse CMEs. Figure 2 shows an inverse and a normal CME launched in the equatorial plane, travelling in the IP space. The shock front travels slower close to the equator resulting in an unusual shape transformation of the CMEs. This is due to the wind model we are using which presents a higher density and a lower velocity at the equator (see Fig. 1). After $9 \mathrm{~h} 36$ min the magnetic cloud still has an almost circular shape. After $55 \mathrm{~h} 11 \mathrm{~min}$ the magnetic cloud is so compressed that it is hard to recognize the Low and Zhang configuration and the CME evolution is far from self-similar. The density redistribution of an inverse and a normal CME is quite similar at $1 \mathrm{AU}$. They are both characterized by a shock front evolving in front of the magnetic cloud which contains the main part of the density. The magnetic field lines are similar for the two CMEs even if the magnetic cloud seems to be more deformed (less circular) for the normal CME.

Figures 3 and 4 show a close-up of these two CMEs just before they reach the Earth. A front shock with an high velocity and relatively high density is clearly visible about $20 R_{\odot}$ before the magnetic cloud (characterized by a very high density blob surrounded by a strong magnetic field). The only real 


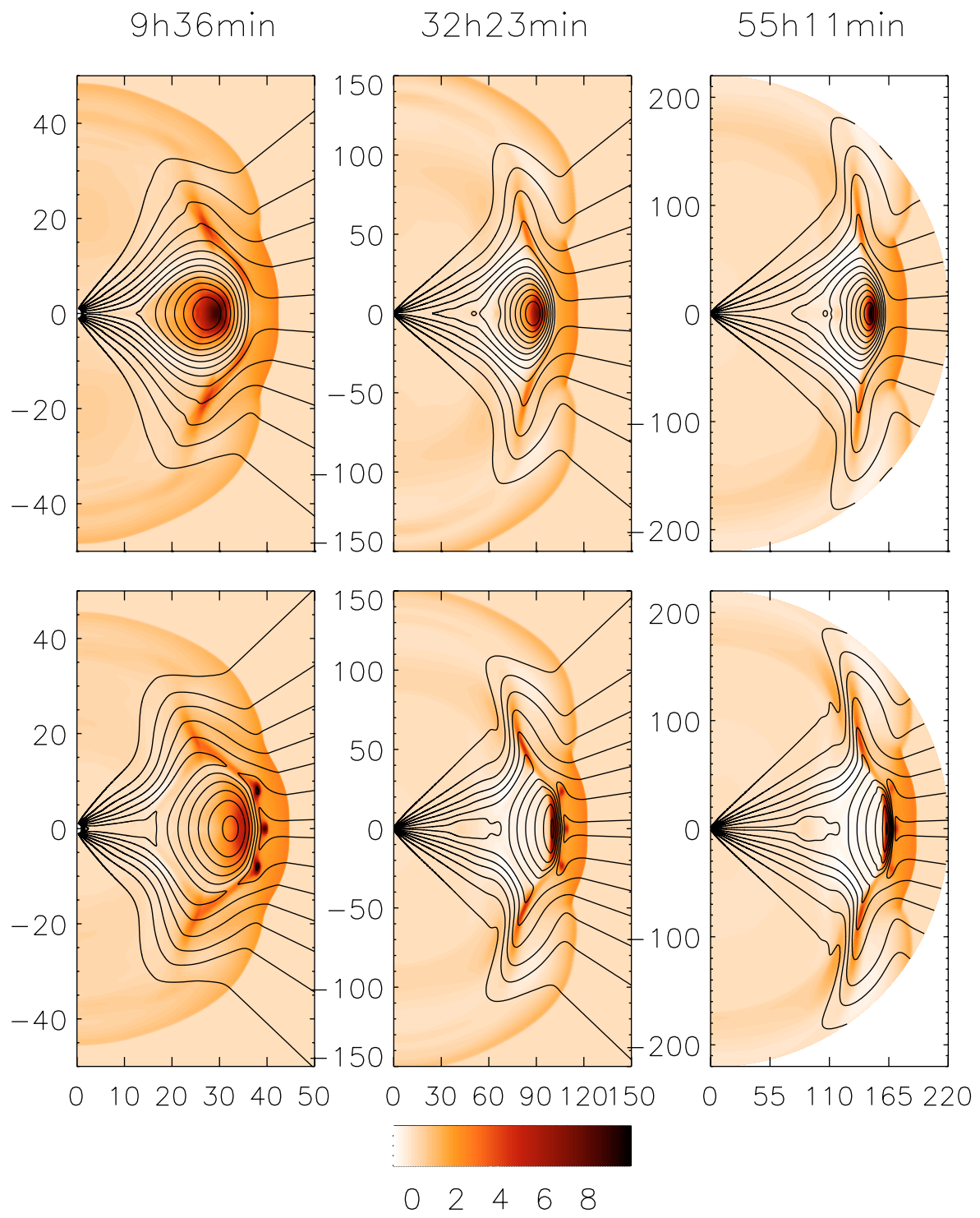

Fig. 2. Contour plots of the relative density distribution for a CME launched in the equatorial plane $\left(\theta_{\mathrm{cme}}=0^{\circ}\right)$. Upper row: inverse initial magnetic polarity, lower row: normal initial magnetic polarity. Left column: after $9 \mathrm{~h} 36 \mathrm{~min}$, central column: after $32 \mathrm{~h} 23 \mathrm{~min}$, right column: after $55 \mathrm{~h} 11 \mathrm{~min}$. The black lines represent the magnetic field. The relative density is define as follow: $\bar{\rho}=$ $\left(\rho-\rho_{\text {wind }}\right) / \rho_{\text {wind }}$. differences between the two CMEs are the orientation of the magnetic field and the fact that the normal CME evolves faster and will thus reach the Earth first. This result is compatible with earlier results, e.g. Zhang \& Low (2004) explain in detail why the normal prominences have a greater expulsion capability.

Figure 5 displays the data obtained by a spacecraft at $1 \mathrm{AU}$ if these CMEs would go through it. One can see that the normal CME shock front arrives at $1 \mathrm{AU} 4 \mathrm{~h}$ before the inverse CME shock front. The inverse CME displays a strong southward magnetic field which may lead to a magnetic storm and the normal CME displays a strong northward magnetic field which normally does not cause a magnetic storm. The velocity of the normal CME is slighty higher and so this CME reaches the Earth a few hours earlier than the inverse CME.

\subsection{Evolution path}

When a CME travels in the IP space, it is important to know its trajectory, i.e. whether its evolution path is a straight line or whether it deviates and by how much. In some cases, this can be crucial information to predict if a CME will hit or miss the Earth. Chané et al. (2005) have studied the evolution path of magnetized CMEs up to $30 R_{\odot}$ and have shown that the initial magnetic polarity of the CME flux rope affects its evolution path substantially: normal CMEs are deviated towards the pole while inverse CMEs are deviated towards the equatorial plane. The magnetic interactions between the background wind and the CME produce two totally different magnetic configurations for an inverse and for a normal CME. Hence the deviation of the CMEs is due to the magnetic pressure which has a different orientation in the two cases. A clear example of this is given in Fig. 7 of Chané et al. (2005). Because of this deviation, the probability of hitting the Earth should be higher for inverse CMEs. We will show that these conclusions remain partially true up to 1 AU. Figure 6 shows the results of several simulations for inverse and normal CMEs launched at $10^{\circ}, 30^{\circ}$ and $60^{\circ}$. For the CMEs at $30^{\circ}$ and $60^{\circ}$, the normal CMEs are deviated towards the pole while the inverse CMEs are deviated towards the equatorial plane. Surprisingly the inverse $\mathrm{CME}$ launched at $10^{\circ}$ is deviated away from the 

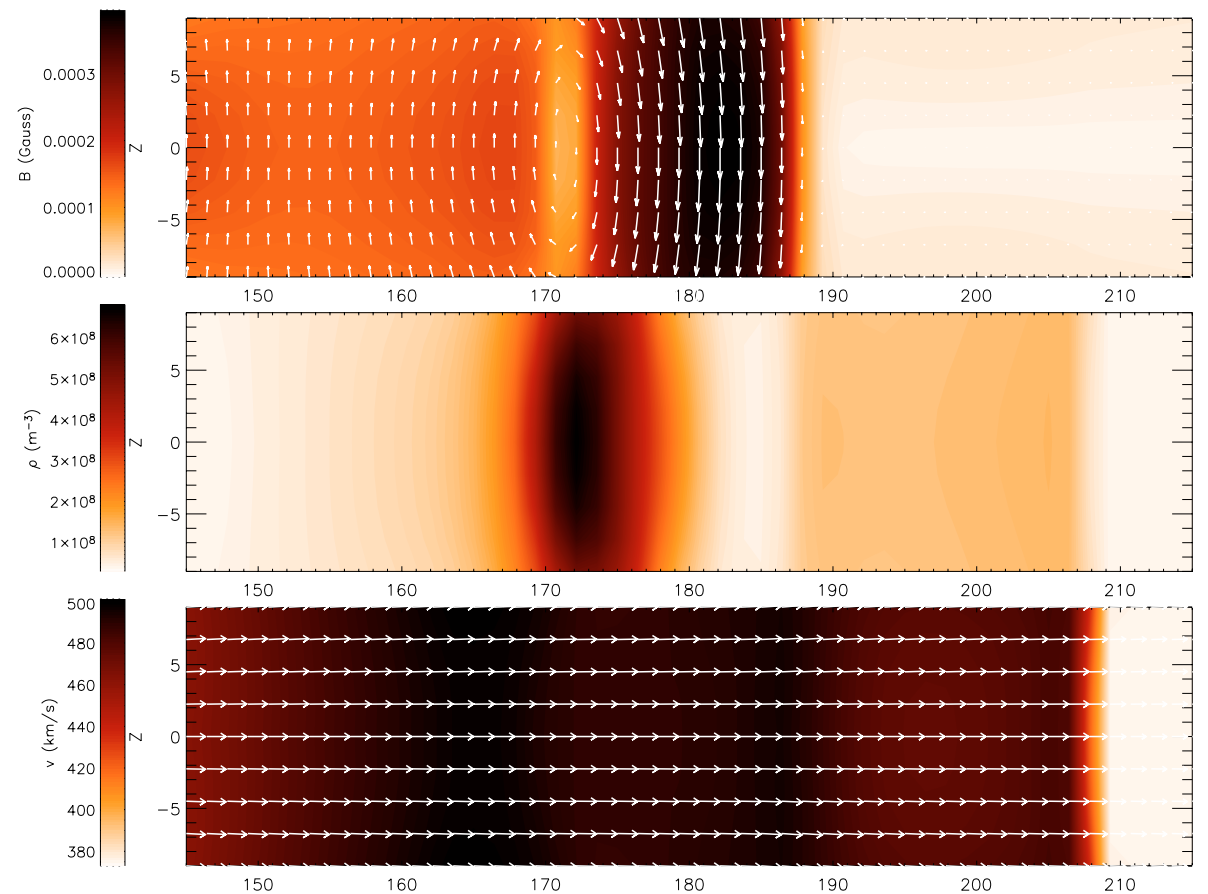

Fig. 3. Contour plot of the magnetic field (Upper row), density (Middle row) and velocity (Lower row) of an inverse CME just before the leading shock reach the Earth (after $64 \mathrm{~h} 44 \mathrm{~min}$ ). The arrows represent the orientation of the magnetic field (Upper row) and of the velocity (Lower row).

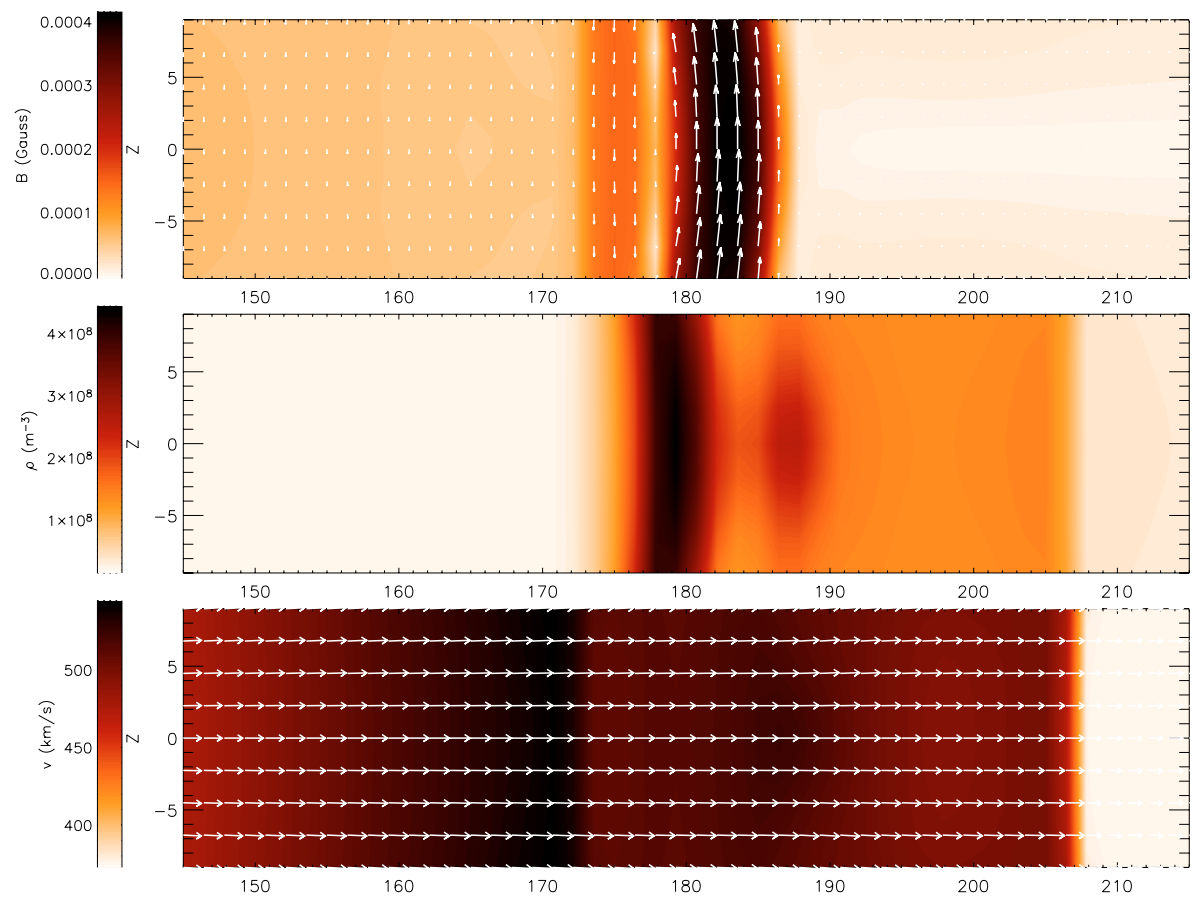

Fig. 4. Contour plot of the magnetic field (Upper row), density (Middle row) and velocity (Lower row) of a normal CME just before the leading shock reach the Earth (after $60 \mathrm{~h} 55 \mathrm{~min}$ ). The arrows represent the orientation of the magnetic field (Upper row) and of the velocity (Lower row).

equatorial plane. This is caused by the density repartition in the wind (see Fig. 1). The higher density close to the equator acts like a natural shield and protects the Earth from the incoming CMEs. However, the density of this wind model is too high in the equatorial plane (see below) and this probably amplifies this phenomenon. Nevertheless, in our simulations, an inverse CME is always travelling closer to the equatorial plane than a normal one and is thus more dangerous for the Earth. 

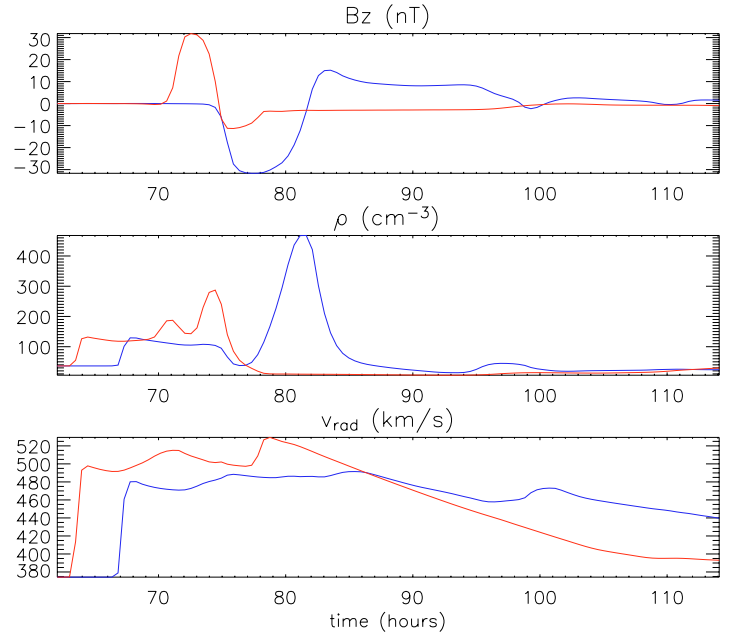

Fig. 5. Evolution of the magnetic field, density and velocity in time in the equatorial plane $\left(\theta=0^{\circ}\right)$ at a position of $215 R_{\odot}$ for a CME launched in the equatorial plane $\left(\theta_{\mathrm{cme}}=0^{\circ}\right)$; red lines: normal CME; blue lines: inverse CME.

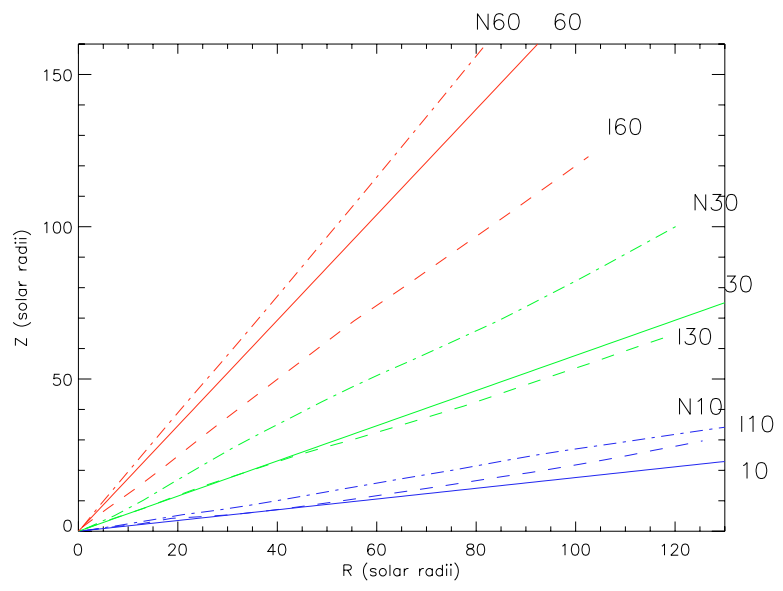

Fig. 6. Evolution path of the center of relative mass for inverse (dashed lines) and normal (dashed-dot lines) CMEs. The CMEs were launched on $10^{\circ}$ (in blue), $30^{\circ}$ (in green), and $60^{\circ}$ (in red). The solid lines show the initial launch angles.

Figure 7 presents data, from our numerical simulations, that would have been recorded by a satellite at 1 AU for CMEs launched at $10^{\circ}$. It is obvious from this figure that the magnetic cloud (an increase of the magnetic field strength followed by an increase of the density) misses the Earth in the case of a normal CME and hits the Earth for the inverse CME. This shows that we need information on the initial polarity of a CME to predict its geo-effectiveness.

\section{In situ data}

On April 4, 2000 a full halo CME event was observed by the Large Angle Spectrometric Coronagraph Experiment (LASCO) and by the Extreme ultraviolet Imaging Telescope (EIT). At 16:32 UT, after a data gap of 90 minutes, the CME was observed for the first time in the $\mathrm{C} 2$ frame. Unfortunately,
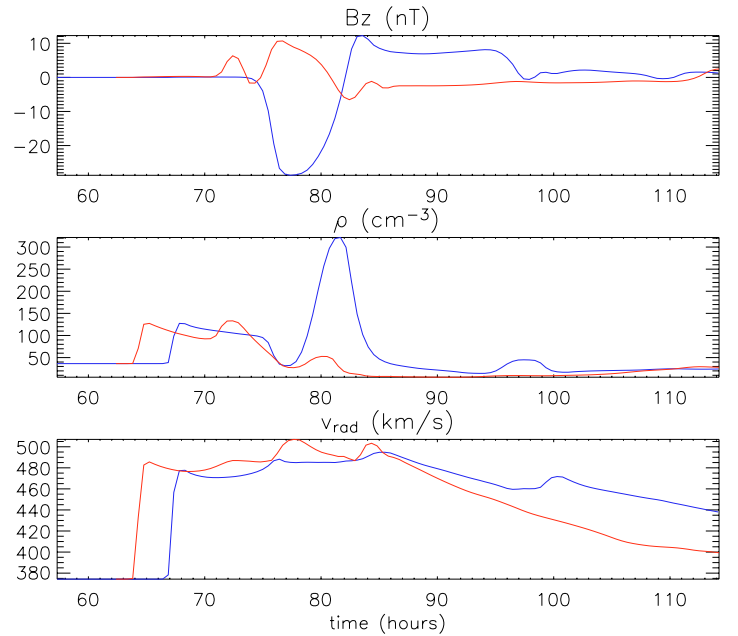

Fig. 7. Evolution of the magnetic field, density and velocity in time in the equatorial plane $\left(\theta=0^{\circ}\right)$ at a position of $215 R_{\odot}$ for a CME launched $\theta_{\mathrm{cme}}=10^{\circ}$ above the equator; red lines: normal CME; blue lines: inverse $\mathrm{CME}$.
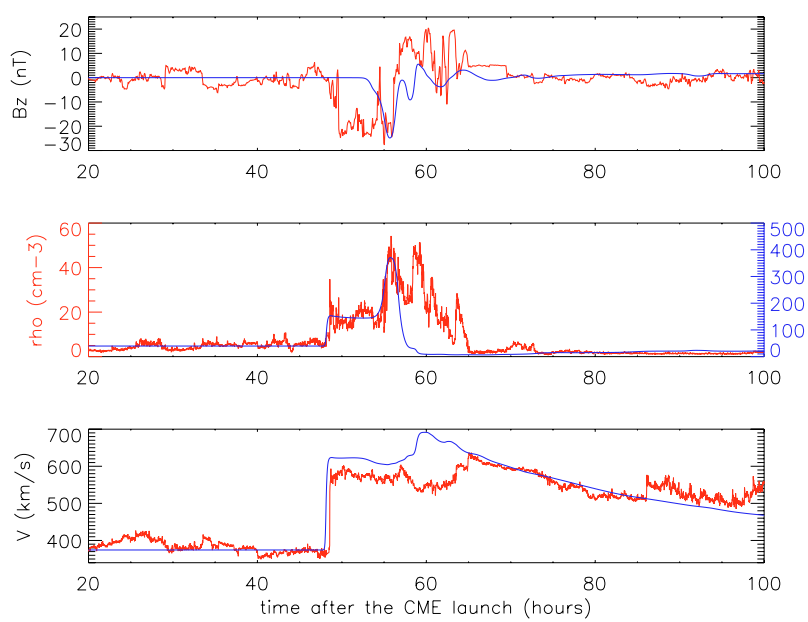

Fig. 8. Comparison between ACE in situ data (red curves) and our simulation (blue curves).

the leading edge of the CME was already out of the C2 field of view (further than $6 R_{\odot}$ ). At 15:24 UT, EIT observed a solar flare probably related to this CME event. According to the C3 measurements, the plane-of-sky speed of the CME was $984 \mathrm{~km} \mathrm{~s}^{-1}$.

We numerically simulate this CME up to $1 \mathrm{AU}$ and compare the measured and simulated signals at $1 \mathrm{AU}$. We use our very simple CME model and assume that at 15:30 UT, the CME was perfectly circular, with an inverse magnetic polarity centered at $1.5 R_{\odot}$ from the Sun center and on the equator. We compare our data at $1 \mathrm{AU}$ with the Advanced Composition Explorer (ACE) spacecraft data. We then adjust our CME parameters (initial speed, initial magnetic strength...) to match the ACE data as well as possible. We thus use the signals close to the Earth to derive the initial characteristics of the CME when it was still close to the Sun. We call this new determination of CME parameters the "a posteriori approach". 

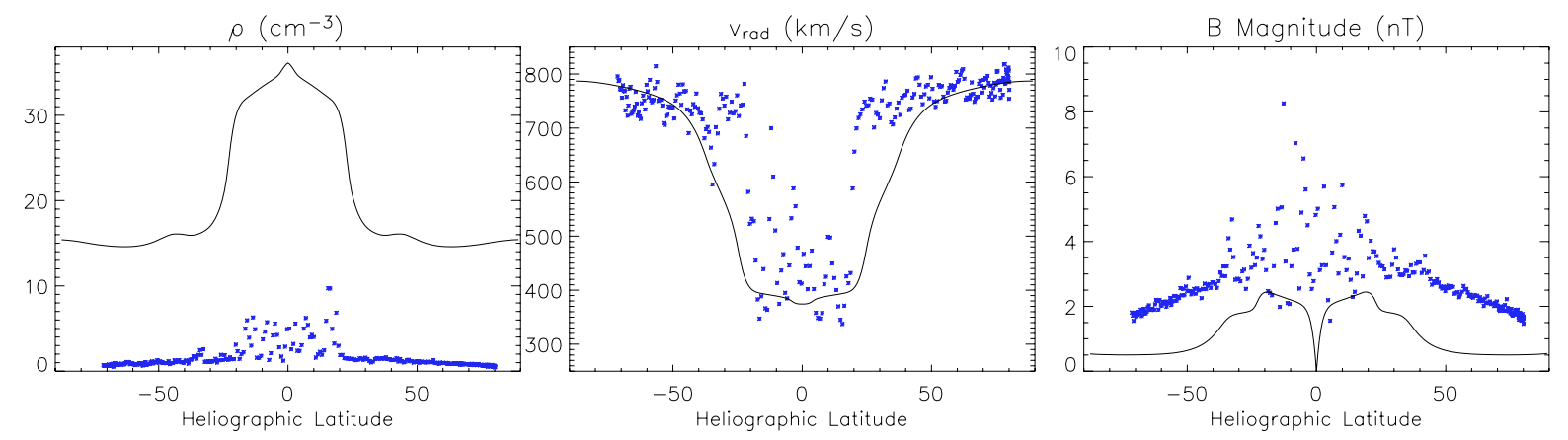

Fig. 9. Comparison between Ulysses data observed between November 1, 1994 and August 1, 1995 when the spacecraft was evolving between 1.34 and $2.03 \mathrm{AU}$ from the sun (blue dots) and the wind model at $1 \mathrm{AU}$ for different latitudes (black curbes).

Figure 8 shows the results of our final best fit. The velocity curve at $1 \mathrm{AU}$ is well reproduced in this simulation. The shape of the density curve also fits quite well, but the values are almost eight times too high. This is probably because of the too high density of the background solar wind we are using (see Sect. 2). From Fig. 9 it is clear that the density in the wind model we applied here is too high (by a factor of more than 20). For the $z$-component of the magnetic field, the simulated and measured profiles at $1 \mathrm{AU}$ are similar but the magnetic cloud in our simulation seems to arrive a few hours too late. This is also related to the wind density: the magnetic cloud is slowed down by the (too) high density of the background wind. Nevertheless the main features of the CME are surprisingly well reproduced in spite of our too simple CME model and that we only performed $2.5 \mathrm{D}$ simulations. To predict the intensity of a magnetic storm, the most important parameter is the z-component of the magnetic field. The solar wind speed is only indirectly important (Wu \& Lepping 2005). We were able to reproduce these two parameters quite well, our model is thus suitable for predictions of the geo-effectiveness of IP CMEs.

According to our simulations, the CME had a maximum initial magnetic field strength of $0.978 \mathrm{G}$, an inverse magnetic field configuration and an initial speed of $1700 \mathrm{~km} \mathrm{~s}^{-1}$ (in the center of the CME). This velocity is about $70 \%$ higher than the one measured. However, this can be explained easily. First, the speed measured by LASCO is the plane-of-sky speed which should be lower than the real CME speed. Second, the $1700 \mathrm{~km} \mathrm{~s}^{-1}$ we mentioned is the initial velocity in the center of the flux rope while the measured $984 \mathrm{~km} \mathrm{~s}^{-1}$ refers to the velocity of the leading edge. Third, because the too high density of the wind slows down the CME, we probably overestimated the initial speed. $1700 \mathrm{~km} \mathrm{~s}^{-1}$ should be considered as an upper limit. There are some papers in the literature that make use of numerical simulations to mimic in situ data (satellite-like curves). See Riley et al. (2002) and Tsurutani et al. (2003) for 2.5D simulations or Manchester et al. (2004b) for 3D simulations. The results did not match very well ACE curves, but none of these papers tried to reproduce one specific CME event.

\section{Discussion and conclusions}

We have extended the study of Chané et al. (2005) on the influence of the initial magnetic polarity on the evolution of CMEs into the IP space by extending the computational domain up to $1 \mathrm{AU}$. We have shown that the evolution path of the CMEs is strongly related to the initial magnetic polarity. An example was given where an inverse CME hits the Earth and where the same CME but with a normal magnetic polarity missed the Earth. We have also shown that the time of arrival is influenced by the initial magnetic polarity: a normal CME propagates faster than an inverse CME and thus reaches the Earth a few hours earlier. The inverse CMEs display a strong southward magnetic field (a well known source of magnetic storms) at $1 \mathrm{AU}$. The geo-effectiveness of a $\mathrm{CME}$ is thus strongly related to its magnetic polarity.

We reproduce the data from the ACE spacecraft after the halo CME on April 4, 2000. By comparing the simulated to the measured data, we deduced the initial characteristics of this CME. In the near future, we plan to improve the wind model in order to improve the fit to the Ulysses data (especially for the density) and to use the "a posteriori approach" with this new wind. The next step in order to have realistic simulations will be to use a 3D model both for the wind and the CMEs.

Our model has the following drawbacks: it is 2.5D; the density of the background wind is too high, i.e. the average solar wind has a much lower density; the CME model we applied is too simple, although it has been used by many other authors. However, in spite of these weaknesses, we obtain a surprisingly good fit to the observations in the case study. Clearly, with a better wind model (e.g. 3D) and a more realistic CME model the data fits should be at least as good and probably better. Will this lead to a different conclusion regarding the initial parameters of the CME? If the conclusions are very similar, this would mean that such complex features for the numerical simulation (e.g. 3D) maybe superfluous. On the other hand, there may be so many free parameters in our model that it is always possible to obtain a good fit with the ACE data.

Acknowledgements. These results were obtained in the framework of the projects GOA 2004/01 and OT 02/57 (KU Leuven), G.0451.05 (FWO-Vlaanderen) and 90203 (ESA Prodex 8). The authors want to thank the National Space Science Data center for the ACE and Ulysses data. The Ulysses data were obtained from the SPDF SOHOWeb database. 


\section{References}

Chané, E., Jacobs, C., van der Holst, B., Poedts, S., \& Kimpe, D. 2005, A\&A, 432, 331

Gombosi, T. I., DeZeeuw, D. L., Groth, C. P. T., Powell, K. G., \& Stout, Q. F. 2000, J. Atmosph. and Terrestr. Phys., 62, 1515

Gopalswamy, N., Barbieri, L., Lu, G., Plunkett, S. P., \& Skoug, R. M. 2005, Geophys. Res. Lett., 32, 3

Groth, C. P. T., De Zeeuw, D. L., Gombosi, T. I., \& Powell, K. G. 2000, J. Geophys. Res., 105, 25053

Jacobs, C., Poedts, S., Van der Holst, B., \& Chané, E. 2005, A\&A, 430, 1099

Keppens, R., \& Goedbloed, J. P. 1999, A\&A, 343, 251

Klimchuk, J. A. 2000, Chapman Conference on Space Weather, AGU, Geophysical Monograph Series 125, ed. P. Song, H. Singer, \& G. Siscoe, 143
Low, B. C., \& Zhang, M. 2002, ApJ, 564, L53

Lugaz, N., Manchester, W. B., \& Gombosi, T. I., in press, ApJ

Manchester, W. B., Gombosi, T. I., Roussev, I., et al. 2004a, J. Geophys. Res., Space Phys., 109, 1102

Manchester, W. B., Gombosi, T. I., Roussev, I., et al. 2004b, J. Geophys. Res., Space Phys., 109, 2107

Riley, P., Linker, J. A., Mikić, Z., et al. 2002, ApJ, 578, 972

Tsurutani, B., Wu, S. T., Zhang, T. X., \& Dryer, M. 2003, A\&A, 412, 293

Vanselow, K. H., \& Ricklefs, K. 2005, J. Sea Res., 53, 319

Wu, C.-C., \& Lepping, R. P. 2005, J. Atmosph. Terrestr. Phys., 67, 283

Zhang, M., \& Low, B. C. 2004, ApJ, 600, 1043 